\title{
Face Detection using Image Morphology - A Review
}

\author{
Venkata Naresh Mandhala ${ }^{1}$, Debnath Bhattacharyya ${ }^{2}$, Tai-hoon Kim $^{3}$ \\ ${ }^{1}$ Department of Computer Science and Engineering, KL University, \\ Vaddeswaram, AP, 522502, India \\ ${ }^{2}$ Department of Information Technology, \\ Bharati Vidyapeeth University College of Engineering, Pune-411043, India \\ ${ }^{3}$ Department of Convergence Security, Sungshin Women's University, \\ 249-1, Dongseon-dong 3-ga, Seoul, 136-742, Korea \\ mvnaresh.mca@gmail.com ${ }^{1}$,debnathb@gmail.com ${ }^{2}$, taihoonn@daum.net ${ }^{3}$
}

(Corresponding Author)

\begin{abstract}
This paper presents an approach by various Algorithms stage by stage for Face Detection. It first detects the face portion, removing all other portion from an image. First it will remove the background and then the body or cloths portion of the image. To achieve this we propose an algorithm based on K-Mean clustering, Bresenham's algorithm, Graham Scan Algorithm. With the help of image morphology, algorithm will detect the skin texture of the face. Image opening and closing will help to get the skin texture. Using a nose template algorithm will find the nose tip by template matching method. Feature vectors are calculated with respect to the nose tip as the origin of 8 octants. Image moment provides a measure for variation in the skin. This total process is done by the feature extraction algorithm.
\end{abstract}

Keywords: K-Means Clustering Algorithm, Bresenham's Algorithm, Graham scan Algorithm, Image Morphology, Template Matching

\section{Introduction}

A face recognition system is a computer application for automatic identification or verification of a person from the digital image of his/her face. One of the ways to do this is by comparing selected facial features from the image and a facial database.

It is typically used in security systems and can be compared with other biometrics such as fingerprint or eye iris recognition systems. Given still or video images of a scene, problem is to identify or verify one or more persons in the scene using a stored database of faces. With the advent of the Internet technology, security is one of the main concern areas for any system. Without using passwords if we use our facial image as a password for the system then system will be more stable in concern of security.

Many algorithms are proposed by researchers to build this system. In this, we have proposed an algorithm which deals with the skin color and texture. Skin texture is nothing but a roughness of the skin of a facial image. This roughness is unique. No two skin texture can have the same texture. Face recognition system has mainly three processes: Face Detection algorithm, Feature Extraction Algorithm, and Face Verification Algorithm. 


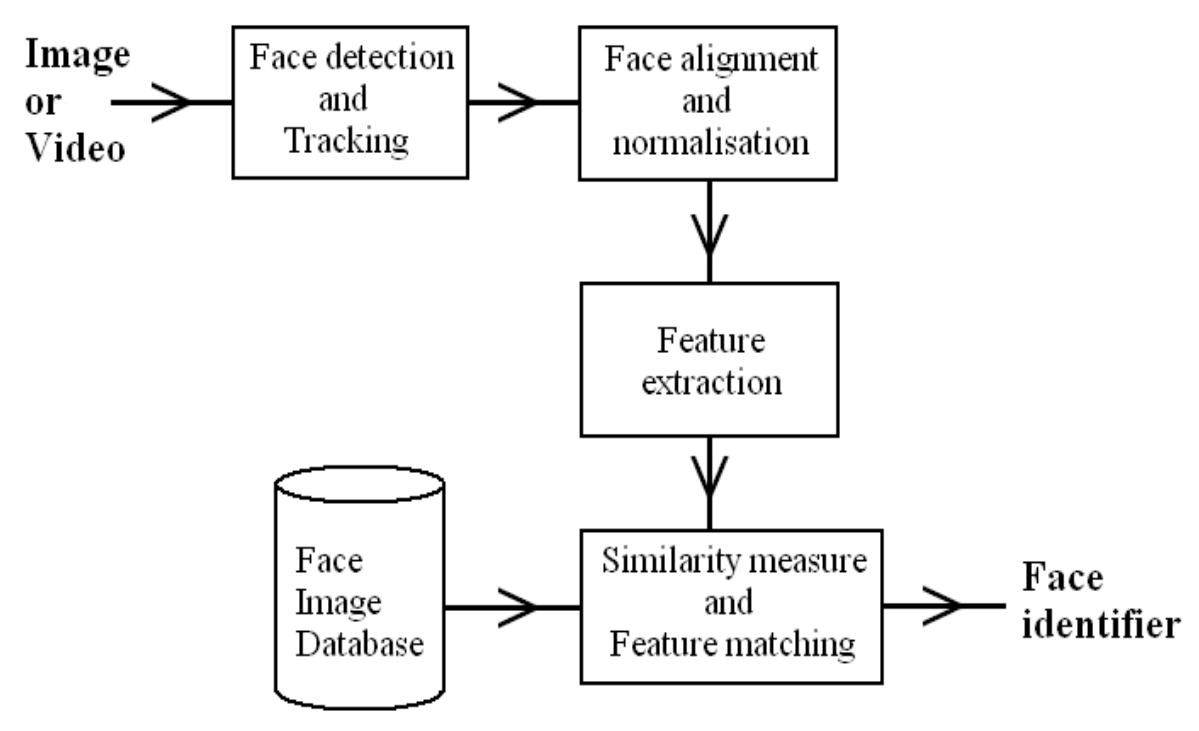

Figure 1. Schematic Diagram of a Face Recognition System

\section{Face Detection}

Face detection module detects or, in other words, separates out human faces from the non-face objects present in an image. The image may be captured either in a controlled environment (probably in a studio or dedicated room with uniform lighting, against a constant background, consisting of a single object, at more or less predefined position, etc.) or in an uncontrolled environment (probably in drawing room, office or even in crowd along with many other objects with various shapes and sizes at various positions with uncontrolled lighting and background). In case of video, the face detected in one frame may be tracked in subsequent frame for acquisition of best view or for activity analysis. An example of face detection is shown in Figure 2.

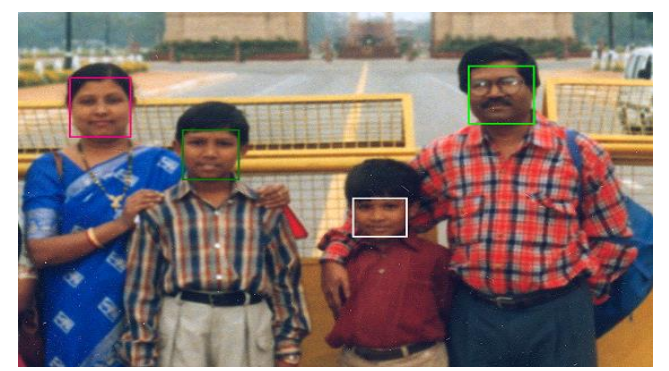

Figure 2. An example of Face Detection

\subsection{Introduction to Face Detection}

Face detection is the first step in automated face recognition. Its reliability has a major influence on the performance and usability of the entire face recognition system. Given a single image an ideal face detector should be able to identify and locate all the present faces regardless of their position, scale, orientation, age and expression. Furthermore, the detection should be irrespective of extraneous illumination conditions and the image content.

Face detection can be performed based on several cues: skin color, facial / head shape, facial appearance or combination of these parameters. Most successful face detection algorithms are appearance based without using other cues. 
In our work we have located or detected a face from a given image by undergoing some different technique step by step.

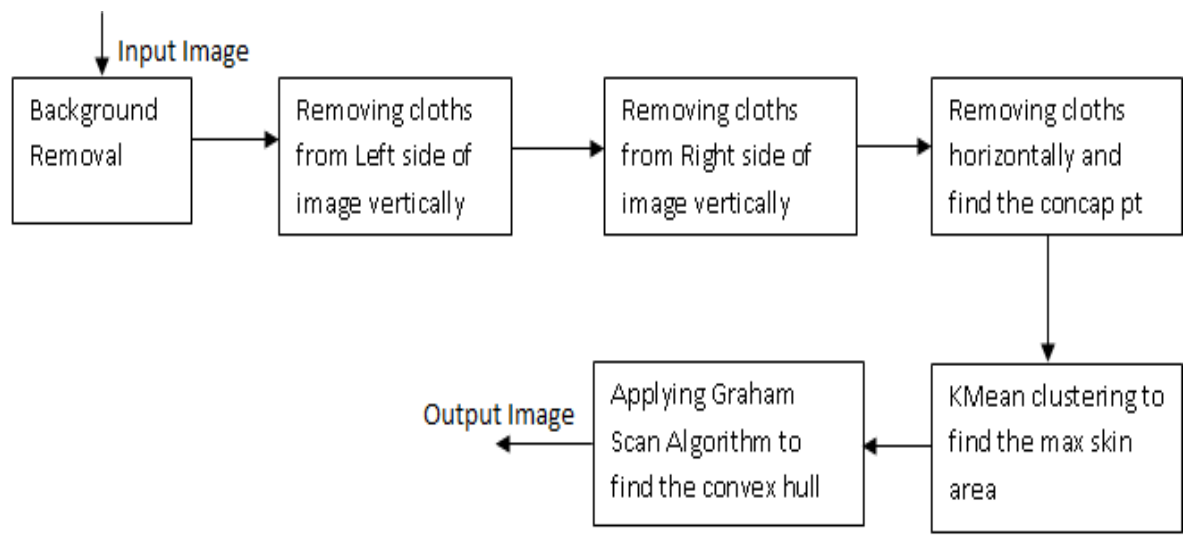

Figure 3. Face Detection Process

Above block diagram is describing the intermediate processes of Face Detection algorithm. Here input image is a sample image and output image is only the detected face area of the input image. Later we will discuss about each process.

\section{Proposed System}

\subsection{Background Removal Process}

In our case sample images have solid background color like red, green, blue etc. The distributions of RGB pixel values are almost uniform. So, if we get the statistical Mean and Standard Deviation (SD) of small sample portion of the background and with the help of multiplying factor of SD we will get the total distribution range of RGB of background pixels.

To achieve this we have taken five sample blocks of the image: 1. Top Left Corner (TLC), 2. Top Middle (TM), 3. Top Right Corner (TRC), 4. Left Middle (LM) and 5.Right Middle (RM). We have calculated Mean and SD of each of these five samples. The multiplying factor is determined by trial and error approach through extensive experimentation.

\subsection{Creating Binary Image}

Binary image has only two pixel values, either 1 (white) or 0 (black). In this case the values will be 255 (white) or 0 (black). Our objective is to create a binary image which will have the face and body potion of the image is black and the background portion is white.

Now we have the image which has no background. We will create a binary image from this image for our further processing. It may happen that when we were removing background, some pixels of face (skin area) and body (cloth area) had been removed. Because of those pixels, value may lie within the range. So we have to get back those pixels to remain the face and body area intact. To achieve this we will create Left Border Binary image and Right Border Binary image. From these two images we will create Binary Image which will have all the face and body portion intact. 


\subsection{Removing Cloths from Binary Image}

After achieving the binary image we will try to remove the cloths from the binary image. If we can remove the cloth portion of the image we will get only the face area of the image. This will be done in four steps. These are the four intermediate processes.

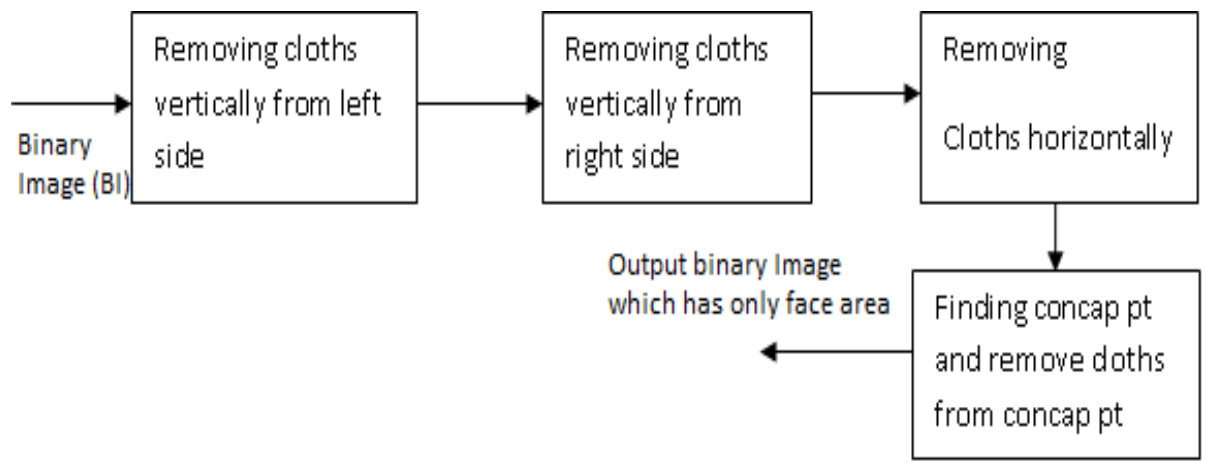

Figure 4. Intermediate Processes of Cloths Removing from Binary Image

\subsection{Finding Concap Point and Removing Cloths}

The concap point lies in between ending of neck and starting of face. It is the conjunction point of neck and face. If we divide black portion of figure 2.3(I') image into two equal half and traverse from middle to left side or right side then the lowest column number of black pixels of all rows will be the concap point.

We have to find out this point. Then only will able to remove all cloths portion successfully. First we will calculate the image (only black portion) height and width. We know the left side vertically cut column number and right side vertically cut column number. From left side cut column we will traverse (left to right) to middle of image width. We also know the horizontally cut row number. From cut row number will traverse from bottom to middle of image height. For both cases we only traverse if we get nonblack pixels. The highest non-black pixel column number of all rows will be the concap point of the image.

From right side of the image we also follow the same technique and also find another concap point. For best result we will take the average of these concap points and remove cloths from this point. It may happen some portion of face of some image may eliminate with the cloths but we will get maximum portion of face.

If we don't find any concap points, leave the image as it is and if we get one concap point then remove cloths from that point.

\section{4. $\mathrm{K}$ - Mean Clustering}

After we achieved the face portion of an image we have to get only skin portion of that image. To do this we have apply K-Mean clustering algorithm on that image. K-Mean clustering algorithm separate different colored pixels and we get the hair portion, skin portion and background portion into three different cluster.

Now challenge is to select the cluster number which has maximum number of skin pixels. To do this we will add all the red component pixels value of every cluster. The cluster number which has max number will be the max skin pixels cluster.

\section{Applying Graham Scan Algorithm}

In selected cluster some pixels has been removed already due to K-Mean clustering. But we need the original face (only skin portion) structure like input image. To achieve 
this we will apply Graham Scan algorithm on the clustered image. This algorithm will find a convex hull of that image. We will get some boundary pixels of that image which cover total area of that image.

Before applying this algorithm we will calculate Mean and SD of that clustered image and we will select those pixels which will lies in between Mean + SD and Mean - SD range. We will do this because after K-Mean clustering may be some portion of hair (other than skin pixels) remains in selected clustered image. To remove those pixels we will do the above process.

\section{Applying Bresenham's Algorithm}

Now we have convex hull points which are nothing but some pixels of clustered image. Applying Bresenham's algorithm we will draw straight lines from one to another. After drawing lines it will look like a polygon. We will fill that polygon by 0 and that filled polygon is the face structure.

\section{Conclusion}

The beauty of proposed system lies in its simplicity, which is not there in other conventional detection system. For most of images it can detect the face. But image quality is the most concerning issue. For bad quality images it may not detect the exact portion of the face. But it will certainly detect some portion of the face, by which we can proceed further.

\section{References}

[1] B. Chanda, D Dutta Majumder, "Digital Image Processing and Applications", Prentice Hall of India (2002).

[2] A. K. Jain, S. Z., Li, "Handbook of face recognition", New York, vol. 1, Springer. (2005).

[3] R. C Gonzalez, S. L.Woods, Eddins, "Digital image processing using MATLAB", Pearson Education India. (2004).

[4] T Kanade., "Picture Processing by Computer Complex and Recognition of Human Faces", Kyoto University, Doctoral dissertation Ph. D. thesis edition. (1973).

[5] A. J. Goldstein, , L. D. Harmon, A. B. Lesk, "Identification of human faces", Proceedings of the IEEE, vol. 59 , no.5, (1971), pp. 748-760.

[6] M. S Kamel, H. C. Shen, A. K. C. Wong, R. I. Campeanu, "System for the recognition of human faces", IBM Systems Journal, vol. 32, no.2, (1993), pp. 307-320.

[7] C. Kotropoulos, A. Tefas, I. Pitas, , "Frontal face authentication using morphological elastic graph matching", IEEE Transactions on Image Processing, vol. 9, no. 4, (2000), pp. 555-560. 
International Journal of Security and Its Applications

Vol. 10, No. 4 (2016) 